\title{
The Informed Consent Process in Genetic Family Studies
}

\author{
LUCY PANOYAN, SHUKO LEE, RAWAN ARAR, HANNA E. ABBOUD \& \\ NEDAL ARAR ${ }^{1}$
}

\begin{abstract}
The informed consent process provides protection by ensuring that potential research subjects understand the goals of the research project they are being asked to voluntarily partake in as well as the risks associated with the study. We examined subjects' comprehension and ability to identify issues explicitly raised during the consent process that was conducted as part of their participation in a genetic family study (GFS). We employed cross-sectional design by providing a short, selfadministrative questionnaire to 246 participants recruited from families enrolled in the Extended Family Investigation of Nephropathy and Diabetes (EFIND) study conducted at the University of Texas Health Science Center. Participants responded to the questionnaire directly after their enrollment in the EFIND study. The questionnaire consisted of multiple-choice questions and focused on the understanding of the purposes, procedures, and risks associated with their participation in the EFIND study. These questions were formulated to reflect basic information presented to subjects through the consent process. Responses to questions were expressed as percentages, placing equal weight on each response. Participants were Mexican-American, $62.3 \%$ female and averaged $35.2 \pm 12.7$ years old (range: 18-76). Our findings showed that the average comprehension score was 58. About $30 \%$ of the participants did not know the name of the study, and $70 \%$ did not identify all elements related to the study procedures. The most striking finding was the lack of understanding concerning the social risks associated with participation in EFIND. While $35.1 \%$ of participants identified all potential physical risks, only $1.3 \%$ could identify all of the social risks. Our findings showed that participants comprehension score was significantly associated with their level of education and income. We conclude that using the informed consent process to communicate research social risks to subjects participating in GFS has some limitations. Future research directed at improving risk communications to subjects of low socioeconomic levels participating in genetic research is justified.
\end{abstract}

\section{Introduction}

In accordance with Federal regulations, no investigator may involve human subjects in research without obtaining informed consent from the subject or the subject's legally authorized representative. ${ }^{2}$ A consent form is a legal document which contains information to help the prospective research subject make an informed and voluntary decision about participation in a study. An informed participant is one who fully understands the research procedure, purpose, and risks of his or her participation. ${ }^{3}$ The informed consent process has been helpful in conducting clinical research; however, in the case of genetic family studies (GFS), researchers face complicated ethical issues. ${ }^{4}$ Genomic research aims to identify genes that may be associated with 
the development and progression of common chronic diseases such as diabetes and associated kidney complications. Using GFS design involves families and populationbased sampling, allowing investigation of both genes and environment, separately or together, and allows valid inference to the wider population. GFS shifts the focus from the traditional individual model to a family model. In the traditional research model, subjects have no ties to other research participants, information learned from the research affects only the subjects, and subjects give their consent to be studied as individuals. By contrast, in the family studies model, subjects have ties to other research participants because of shared genetic heritage. In addition, information learned from the research affects the whole family, and other family members unwillingly become part of the study without their consent. ${ }^{5}$

There are, however, ethical challenges in ensuring adequate human subject protection and addressing participant concerns regarding such research. The use of an individual's genetic information has the potential to affect negatively not only the individuals and their families, but also population groups with which the subject is associated. ${ }^{6}$ When the sample is identifiable, there is concern that the donor's privacy will be violated ${ }^{7}$ leading to the scrutiny, prejudice, coercion and judgment of others and loss of insurability and employment. ${ }^{8}$ If the sample is unidentifiable, there is a possibility that a subject's specimen could be used for future research, which the donor finds objectionable due to social or religious norms within his or her subculture. Social repercussions of GFS also put racial and ethnic groups at risk due to the potentially stigmatization of specific populations. ${ }^{9}$ Additionally, in pedigree studies, non-paternity and non-maternity may also be unexpectedly revealed, altering family relationships evermore. Because of these social, economic, legal, and psychological ethical challenges, ${ }^{10}$ communicating research on social risks and ethical issues to enrolled subjects is vital to ensure informed and voluntary participation. ${ }^{11}$ Not much is known about the utility of the informed consent process in genetic family studies. We examined subjects' comprehension of information related to their participation in GFS as presented to them through the consent form.

\section{Methods}

\section{Subjects and Procedures}

Participants were recruited from families enrolled in the EFIND study conducted at the University of Texas Health Science Center (UTHSCSA). The EFIND study aimed to establish complex pedigrees by enrolling extended family members that participated in the initial FIND study to identify gene(s) involved in the development and progression of diabetic nephropathy (DN) in Mexican-Americans (MA). The EFIND study inclusion criteria entail recruiting probands who have advanced DN and have both parents living and willing to participate in the study and/or at least one living sibling with type 2 diabetes mellitus (T2DM) willing to participate in the study. Siblings that are either "concordant" (diagnosed with DN) or "discordant" (diagnosed with T2DM for $10+$ years without DN) are enrolled. A total of 246 subjects were enrolled in EFIND.

In this paper, we report the results obtained from subjects participating in the EFIND study to assess subjects' understanding of the study's protocol, and of the risks 
associated with their participation. After informed consent was obtained from all participants, blood and urine samples were collected to measure clinical phenotypes and to isolate DNA for genetic analysis. The process of obtaining informed consent lasted for an average of 35 minutes, and was performed by a trained study coordinator experienced in conducting GFS. The consent form was seven-pages long and read at a 6th-grade level. Subjects were given the form to read, and subsequently the study coordinator actively reviewed all of the information and answered participants' questions. The study staff specifically presented information to participants such as how confidentiality and privacy will be protected. In addition, they emphasized that all information about subjects' DNA, blood and urine samples, and cell culture will be kept confidential and will be assigned an identification number. The study staff also asked subjects about their preferences concerning disclosure of the study results. The consent form of the EFIND study included multiple-choice questions asking participants about their preferences in (1) receiving their lab results, (2) receiving their genetic results, and (3) participation in future research.

All participants were offered a short self-administrative structured questionnaire directly after their enrollment in the EFIND study. The purpose of the questionnaire was to determine how much information participants have comprehended about the study's protocol, and about the physical and social risks as described in the consent form. We selected to offer the questionnaire directly after enrollment in order to minimize the lag time between the information provided to them during the consent process and their responses to our questions. The questionnaire was previously test piloted using 30 subjects and revised accordingly. The multiple choice questions were formulated to reflect basic information presented to subjects through the consent process. Choices for each question included sentences simply extracted from the consent form with minor rewording. Six questions were intended to test subjects' understanding as it relates to the purpose and procedures of the study. Three questions addressed potential physical and social risks associated with GFS. Two questions assessed subjects' perception about the purpose of the consent form. In addition, the questionnaire gathered data on basic demographic information such as level of education, income, age, and gender. Subjects' responses to questions about personal health information and voluntary participation are not part of this report. These responses focused on different aspects of the study and will be presented in future publications.

\section{Study Design and Procedures}

We employed a cross-sectional study design. On average, administering the questionnaire required about 15 minutes; it was conducted in English or Spanish according to participants' individual preferences. Subjects had no major health impairment that would impede their participation. The Institutional Review Board at UTHSCSA approved the study protocol.

Study variables included subjects' (1) demographic characteristics such as gender, age, level of education and income, (2) responses to EFIND study goals and procedures, (3) response to the physical and social risks and (4) responses to the purpose of the consent form. The following is a brief description of our approach for collecting and analyzing data related to these variables. 


\section{Data analysis}

Respondents were asked to circle all valid responses. Responses to questions were expressed as percentages with equal weight given to each answer choice. The average score for each subject was calculated. For example, one question asked participants to identify all of the physical risks associated with the study based on what they learned during the consent process. The answer choices were the following:
a) bleeding and bruising at the site were blood was drawn,
b) temporary, local discomfort that accompanies all needle sticks,
c) infection at the site where blood was drawn,
d) dizziness when the blood sample is taken, but this is temporary and mild, and
e) I do not know.

To receive a complete score (100), participants had to identify all four physical risks. Each question was scored individually in this manner, and then the averages were combined to represent the "average comprehension score" for the questionnaire. Descriptive statistics such as frequency distribution and means were applied to analyze categorized and continuous variables. Associations between categorical variables in the form of contingency tables were examined using a $\chi^{2}$-test. Correlation between the average comprehension score and some selected demographic variables were also tested. The software package SPSS (V9) was used for analyzing quantitative data.

\section{Results}

Of the 246 participants enrolled in the EFIND study, 61\% $(n=151)$ participated in the self-administrative questionnaire. Participants were $62.3 \%$ female and averaged 35.2 \pm 12.7 years old (range: $18-76$ ). About $66 \%$ of participants were not formally educated beyond high school, $24.5 \%$ reported a household income $<\$ 20,000$.

\section{Responses to EFIND study goals and procedures}

(See Table I) About seventy percent $(70.9 \%)$ of the participants identified the name of the study, while about one-third of the subjects could not. When asked about the goals of the study, $17.3 \%$ identified both goals: the genetic and ethical aims. Eighty percent $(80 \%)$ indicated that the study was aimed at examining the genetics of diabetes, and $2.7 \%$ did not know.

Subjects were asked to choose all items related to the study's procedure: (1) an interview to review medical history and establish a family tree, $(2)$ review medical records, (3) draw blood, (4) collect a urine sample and (5) obtain blood pressure, height and weight.

About thirty percent (31.8\%) of the participants identified all five items of the study procedures. About twenty percent (19.9\%) identified four items, 23.2\% identified three items, and $10.6 \%$ identified two items. About $14 \%$ identified one item. 


\begin{tabular}{l|c}
\hline & Frequency $(\%)$ \\
& $N=151$ \\
\hline Name of Study: & $107(70.8)$ \\
Identified the name of the study & $44(29.3)$ \\
Did not know the name of the study & \\
Purpose of Study: & $26(17.3)$ \\
Identified both purposes & $120(80)$ \\
Identified one purpose & $4(2.7)$ \\
Did not know & \\
Study Procedures: & $48(31.8)$ \\
Identified all procedures & $30(19.9)$ \\
Identified 4/5 procedures & $35(23.2)$ \\
Identified 3/5 procedures & $16(10.6)$ \\
Identified 2/5 procedures & \\
Physical Risks: & $53(35.1)$ \\
Identified all physical risks & $11(7.3)$ \\
Identified 3/4 physical risks & $21(13.9)$ \\
Identified 2/4 physical risks & $54(35.8)$ \\
Identified $1 / 4$ physical risks & $12(7.9)$ \\
Did not know physical risks & \\
Social Risks: & $2(1.3)$ \\
Identified all social risks & $21(14)$ \\
Identified 3/4 social risks & $16(10.7)$ \\
Identified 2/4 social risks & $80(53.3)$ \\
Identified $1 / 4$ social risks & $31(20.7)$ \\
Did not know social risks &
\end{tabular}

Table I: Subjects' understanding of the study protocol, physical and social risks

Response to the physical and social risks

(See Table I) Participants were asked to choose all possible physical risks associated with their participation based on their understanding of the materials presented to them through the consent form. Potential physical risks included:

(1) bleeding and bruising at the site where your blood was drawn.

(2) temporary, local discomfort that accompanies all needle sticks.

(3) infection at the site where blood was drawn.

(4) dizziness when the blood sample is taken.

About one third of the participants (35.1\%), identified all four potential physical risks compared to $35.8 \%$ who identified only one item. About eight percent $(7.3 \%)$ recognized three items, and $13.9 \%$ chose two items.

Participants were also asked to list all possible social risks associated with their participation in GFS based on information presented to them through the consent form. These social risks are the following:

(1) Genetic analysis may reveal whether or not the parent of a child is the actual biological parent.

(2) There is no way to ensure absolute protection of the privacy of genetic information. 
(3) Anxiety may occur from knowing of an increased risk for kidney disease or diabetes.

(4) Discrimination by health insurers and employers may occur.

Participants' responses were striking. Only $1.3 \%$ of subjects identified all four social risks. Whereas $53.3 \%$ identified one risk, $14 \%$ chose three items, and $10.7 \%$ of the subjects selected two items. Twenty percent $(20.7 \%)$ said they do not know.

\begin{tabular}{l|l}
\hline & Frequency (\%) \\
\hline Purpose of Consent Form: & \\
Identified both purposes & $61(40.4)$ \\
Thought purpose was to understand goals/procedures/risks and & $45(29.8)$ \\
benefits & \\
Thought purpose was to ensure voluntary participation & $42(27.8)$ \\
Did not know purposes & $3(1.99)$ \\
When to Sign Consent Form: & $49(32.5)$ \\
Identified all reasons & $5(3.3)$ \\
Identified 3/4 reasons & $15(9.9)$ \\
Identified 2/4 reasons & $73(48.4)$ \\
Identified 1/4 reasons & $9(5.9)$ \\
Did not know reasons & \\
\hline
\end{tabular}

Table 2: Subjects' responses regarding purpose of the consent form

Responses to the purpose of the consent form

(See Table 2) Participants were asked about the purpose of the consent form and when they should sign it. Forty percent $(40.4 \%)$ of the subjects indicated that the purpose of the consent form was to understand the study protocol and to ensure voluntary participation. Fifty seven percent (57.6\%) identified one purpose, and about $2 \%$ did not know. Four reasons were listed as to why participants should sign the consent form:

(1) To decide to take part in this research study.

(2) To authorize the collection, use and disclosure of protected health information.

(3) To reiterate that all questions about the study have been answered.

(4) To understand all of the information given about the study, the use, and the disclosure of health information.

Of the four reasons, the item "decided to take part in the study" was chosen with the highest frequency (48\%). This indicated that most participants believed that they should sign the consent form if they agreed to take part of the study. Thirty two percent $(32.5 \%)$ responded to all four items, three percent $(3.3 \%)$ identified three items and, nine percent $(9.9 \%)$ recognized two items.

Our findings showed that participants' average comprehension scores (58, range: 16100) were significantly associated with their level of education $(r=0.2216, p=0.0062)$ and income $(\mathrm{r}=0.2214, \mathrm{p}=0.0156)$. There were no significant correlations between age or gender with the total average comprehension scores. 


\section{Discussion}

Examination of the approach used for protecting human subjects participating in genetic research has highlighted concerns about its overall effectiveness. We assessed subjects' comprehension of the study protocol, and of risks associated with their participation in the EFIND study. Our findings revealed an average comprehension score of 58, indicating that some participants gave their consent without fully understanding the study protocol and risks. Similarly, in a systematic review, Cohen and Larson ${ }^{12}$ suggested that despite increasing regulatory scrutiny, deficiencies still exist in participants' comprehension of the research in which they participate. In our study, we found a strong correlation between educational levels and subjects' average comprehension score. Likewise, previous studies showed that research participants with higher education or reading levels had significantly higher understanding scores. The differences in understanding due to education were often large, especially compared with the improvements produced by the various interventions. For example, Taub and Baker ${ }^{13}$ documented that a feedback intervention increased average understanding scores by $29 \%$, but there was a $95 \%$ difference between the least educated quartile of research participants and the most educated quartile. However, they also acknowledged that participants' recall of the information presented to them during the interview may not always reflect their actual understanding of such information.

The strong correlation between the level of education and participants' comprehension score is not surprising. Enrolling subjects with a low educational level and ensuring a reasonable understanding of the study protocol and risks poses a real challenge. In conducting genetic family research over the last decade, we found that most subjects were willing to participate in these studies to help researchers and advance science. However, high proportions of our research subjects reported a low level of education. ${ }^{14}$ Future studies directed at improving subjects' understanding of risks and ethical issues associated with their participation in genetic research is highly recommended.

The most striking finding in our work is the lack of understanding of the social risks associated with the GFS. While $35.1 \%$ of participants identified all potential physical risks, only less than $2 \%$ could identify all of the social risks. These findings present important implications to obtaining informed consent from subjects participating in GFS. Unlike research in which the risks are primarily physical, GFS poses greater social risks. The main physical risk in GFS, the drawing of blood, is negligible. However, the potential magnitude of social, economic, legal, and psychological harms is exceedingly greater. The social and ethical concerns associated with genetic research are complicated and pose a real risk for individuals participating in the study. ${ }^{15}$ As a result of a survey conducted in 1995 involving people with a known genetic condition in their family, $22 \%$ of the participants were denied health insurance because of their health status. This denial only represents the reported number and does not take into consideration whether or not the participant was already sick. ${ }^{16}$ According to the second annual Cogent Research Syndicated Genomics Attitudes \& Trends (CGAT) Survey conducted by Cogent Research in Cambridge, Massachusetts 
in $2005,{ }^{17}$ the public's greatest concern about genetic testing addresses privacy issues. This includes the fear that genetic information will lead to discrimination ${ }^{18}$.

The IRB requires that consent forms be written at the 6th-grade level, making it possible for the participant to understand the scientific aspects associated with the study. This becomes more complicated with genetic studies because genetic terminology is difficult to phrase in layman's terms ${ }^{19}$. According to the CGAT survey, there is small number of people who fully understand the concept of genomics. Five percent of those who have heard or read something about genomics can explain that genetic testing can facilitate early diagnosis. Three percent noted that genomics can help customize treatments. This indicates that there is a lack of understanding when it comes to genetics ${ }^{20}$.

Achieving effective informed consent participation remains a major challenge for subjects participating in GFS. Several efforts have been directed to improve participants' understanding of information disclosed in the informed consent process. $^{21}$ Some interventions have focused on the use of multimedia. ${ }^{22}$ Others have enhanced the consent form by condensing its length, revising its content, improving formatting, and adding graphics. ${ }^{23}$ Only a few interventions have been directed at improving participants' comprehension of risks and ethical issues associated with their participation in genetic research. Previously, we as well as others have suggested that having a study team member or a neutral educator spend more time talking oneon-one to study participants appears to be effective way of improving the understanding of subjects regarding the research protocol. ${ }^{24}$ We proposed a "subjectcentered approach" that viewed enrollment as an active process in which subjects and recruiters communicate on risks and ethical issues related to subjects participating in genetic research. ${ }^{25}$ Another way to address the complexity of presenting social risks to subjects participating in GFS is to provide visual representations of these risks. Educational aids may include the use of charts, models, DVDs, videotapes, and other audio-visual presentations that may assist in communicating the procedures and risks. In addition, a semi-tutorial session explaining basic concept of the genetics involved with the study could be more effective than reading a consent form alone. For complex protocols, the incorporation of diagrams and flow charts into the consent document itself may enhance clarifications of the research procedures.

Initiatives to better safeguard the rights and welfare of research subjects participating in genetic research require a broader vision. Research volunteers should accurately and effectively be informed about what they are getting into. ${ }^{26}$ Improving the content of the consent form is only one aspect of this endeavor, but addressing the complex ethical and social implications is the ultimate goal $^{27}$ for future research in this important area.

One potential limitation of this work is the ability to generalize the findings to broader groups of multi-ethnic origins. Our intention in this article was not to generalize the current findings, but rather to present and describe how a group of low-income Mexican-Americans comprehends information about their participation in genetic research. Although the broader implications of our findings remain to be 
demonstrated, we believe they will prove to be especially useful for improving the consent process among low-income minority Mexican-American populations.

\section{Acknowledgements}

We wish to thank all participants of the EFIND Study for their cooperation and generous participation. This study was supported by funds from NIH/NIDDK (P50 DK061597). This work was also supported by the VHA-HSR\&D (PI: Nedal Arar). The views expressed in this article are those of the authors and do not necessarily represent the views of the Department of Veterans Affairs. The Institution of Integrating Medicine and Science (IIMS) at UTHSCSA provided clinical support for this project.

\footnotetext{
${ }^{1}$ All the authors are at the Division of Nephrology/Department of Medicine, University of Texas Health Science Center at San Antonio, San Antonio, Texas, USA, and the Audie L. Murphy Memorial Veterans Hospital, San Antonio. Corresponding author: Nedal Arar ararn@uthscsa.edu

${ }^{2}$ UNM Health Sciences Center. Manual for Conducting Human Subject Research Informed Consent Requirements. New Mexico, 2006.

${ }^{3}$ National Commission for the Protection of Human Subjects of Biomedical and Behavioral Research. 1979. The Belmont Report: Ethical Principles and Guidelines for the Protection of Human Subjects of Research. Washington, DC.

${ }^{4}$ H. Allen. Genetic protocols review by Institutional Review Boards at National Cancer Institutedesignated cancer centers. Genetic Testing 1998; 2(4):329-334; American Society of Human Genetics. Eugenics and the misuse of genetic information to restrict reproductive freedom. American Journal of Human Genetics 1999; 64:335-338; L. Andrews. Compromised Consent: deficiencies in the consent process for genetic testing. Journal of the American Medical Women's Association 1997; 52(1): 39-42, 44; G.J. Annas. Reforming informed consent to genetic research. Journal of the American Medical Association 2001; 286(18): 2326-2328.

${ }^{5}$ R. Bennet. 1999. The Practical Guide to the Genetic Family History. New York: Wiley-Liss Publications.

${ }^{6}$ Ibid; M.A. Rothstein. Expanding the ethical analysis of biobanks. Journal of Law, Medicine \& Ethics 2005; 33(1): 89-101.

${ }^{7}$ B.R. Bates, J.A. Lynch, J.L. Bevan, C.M. Condit. Warranted concerns, warranted outlooks: a focus group study of public understandings of genetic research. Social Science \& Medicine 2005; 60:331344.

${ }^{8}$ J.W. DeCew. Privacy and policy for genetic research. Ethics and Information Technology 2004; 6:514.

${ }^{9}$ Rothstein, op. cit. note 6 .

${ }^{10}$ Bennet, op. cit., note 5; Rothstein, op. cit. note 6; Bates, op. cit. note 7; DeCew, op. cit., note 8.

${ }^{11}$ N.H. Arar, H. Hazuda, R. Steinbach, M.Y. Arar \& H.E. Abboud. Ethical issues associated with conducting genetic family studies of complex diseases. Annals of Epidemiology 2004; 15(9): 712-719.

${ }^{12}$ E. Cohn \& E. Larson. Improving participant comprehension in the informed consent process. Journal of Nursing Scholarship 2007; 39(3): 273-80

${ }^{13}$ H.A. Taub, M.T. Baker. The effect of repeated testing upon comprehension of informed consent materials by elderly volunteers. Experimental Aging Research 1983; 9: 135-138.

${ }^{14}$ N.H. Arar, R. Plaetke, B. Kasinath \& H. Abboud. Incorporating Contextual Assessment Approach into Regimens Used In Genetic Family Studies. Genetics in Medicine 2002; 4(6): 451-63.

${ }^{15}$ Allen, op. cit. note 4.

${ }^{16}$ M. Simon. Genetic Discrimination: Myth or Reality? Genetic Health Nov. 2000; http://genetichealth.com/ELSI_Genetic_Discrimination_Myth_or_Reality.shtml (accessed 9 December 2006)
} 
${ }^{17}$ C. White, J. Meunier \& G. Steelfisher. Public Perception of Genomics/Genetic Testing: CGAT Survey Results. Pharma Week 2007

${ }^{18}$ S. Spotswood. VA Ponders Genomics As Treatment Avenue. US medicine July 2006

(http://www.usmedicine.com/article.cfm?articleID=1344\&issueID=89)

${ }^{19}$ N. King. Rewriting the "Points to Consider": The Ethical Impact of Guidance Document Language. Human Gene Therapy 1999; 10: 1333-139.

${ }^{20}$ White, op. cit., note 17; http://www.pharmaweek.com/Exclusive Content/11_4.asp

${ }^{21}$ T.C. Davis, R.F. Holcombe, H.J. Berkel et al. Informed consent for clinical trials: a comparative study of standard versus simplified forms. Journal of the National Cancer Institute 1998; 90: 668-674.

${ }^{22}$ P. Agre, F.A. Campbell, B.D. Goldman et al. Improving informed consent: the medium is not the message. IRB 2003; 25: S11-S19

${ }^{23} \mathrm{~J}$. Flory \& E. Emanuel. Interventions to improve research participants' understanding in informed consent for research: a systematic review. JAMA 2004; 292(13): 1593-601

24 J.R. Botkin. Informed Consent for Genetic Research. Current Protocols in Human Genetics 2005;

1.16.1-1.16.12

25 Arar, op. cit. note 11.

${ }^{26}$ Taub, op. cit. note 13 .

${ }^{27}$ L.M. Beskow, W. Burke, J.F. Merz et al. Informed Consent for Population-Based Research Involving Genetics. JAMA 2001; 286(23): 15-2321; C.A. McCarty, A. Nair, D.M. Austin \& P.F. Giampietro. Informed consent and subject motivation to participate in a large, population-based genomics study: the Marshfield Clinic Personalized Medicine Research Project. Community Genetics 2007; 10(1): 2-9. 